\title{
LA RELACIÓN ACCIONISTA-EMPRESA EN INTERNET ANÁLISIS DESDE EL ENFOQUE DE CONFIANZA
}

\author{
María del Mar Alonso Almeida \\ Universidad Autónoma de Madrid \\ mar.alonso@uam.es
}

\begin{abstract}
Resumen
Durante la última década se ha producido una ruptura de la confianza de los accionistas hacia las empresas. Son varios los factores que han contribuido a propiciar esta ruptura; entre ellos determinados abusos de la «contabilidad creativa» reflejados en un excesivo «maquillaje» de la información económico-financiera y las malas prácticas de algunos agentes de los mercados que terminan por originar desconfianza e incertidumbre acerca del presente y las perspectivas de desarrollo del sistema económico en el futuro. El objetivo de este trabajo es analizar las bases de la confianza entre accionistas y empresas y proporcionar un modelo de comunicación a través de Internet que contribuya a construir la confianza entre ambos.
\end{abstract}

Palabras clave: confianza, accionista, Internet, código de buen gobierno.

\begin{abstract}
During the last decade there has been a break of trust of the shareholders to the company. Several factors converge to bring this breakthrough on the one hand, certain abuses of the "creative" reflected in an excessive "make-up" of the financial information and on the other hand, poor practices among some market players that have resulted in distrust and uncertainty about the present and the prospects for future. The aim of this paper is analyze trust between shareholders and companies and provide a model of communication via Internet to contribute to build confidence between them.
\end{abstract}

Key words: trust, shareholder, Internet, governance codes. 


\section{Introducción}

La investigación sobre ciencias sociales da cada vez mayor importancia a la existencia de confianza en las relaciones sociales, económicas, políticas, legales y organizacionales, como lo demuestra el cada vez mayor número de investigaciones que se han realizado con este enfoque (Garment, 1991; Miller, 1992; Putnam, 1994; Sitkin y Roth, 1993, Bianco, 1994; Brown, 1994; Cortina et ál., 2003; Alonso Almeida, 2007).

Es muy difícil concebir la vida económica sin un nivel mínimo de confianza, como explicó Kenneth Arrow, premio Nobel de Economía:

...la confianza adquiere, como mínimo, un valor pragmático muy importante, es un significativo lubricante del sistema social; es sumamente eficiente: se ahorra uno muchos problemas cuando confía en la palabra de los demás. Por desgracia, ésta no es una mercancía que se pueda comprar con facilidad. La confianza y otros valores similares son ejemplos de lo que los economistas llaman externalidades, son bienes, tienen un valor real práctico y económico, incrementan la eficiencia del sistema y permiten producir más bienes o mayor cantidad de cualquier valor apreciado (1974: 84).

Para entender el valor económico de la confianza se puede examinar cómo sería un mundo carente de ella y todos los costos asociados a esta situación (Fukuyama, 1998). Por tanto, se requiere confianza para todas las relaciones que se producen entre empresa y sociedad (Putnam, 2000). Pero no solo constituye un lubricante o facilitador de las relaciones sino que es la base primera para cualquier tipo de interacción y actúa reduciendo la complejidad con mayor rapidez y más economía que las predicciones del mercado, los reguladores o las negociaciones. Sin embargo, para que se produzca esa primera interacción entre agentes y organizaciones es necesario que se dé como condición de partida la presencia de relaciones sociales armoniosas y la inexistencia de conflictos. De acuerdo con los estudios realizados, cuando esto se consigue se comprueba que un comportamiento organizacional basado en la confianza reduce los costos de transacción.

A continuación se exponen las bases teóricas de la confianza y cómo se construye esta, luego se explica la manera en la cual Internet puede servir de canal para generar la confianza necesaria entre una empresa y sus accionistas.

\section{Fundamentos teóricos de la confianza}

La doctrina ha definido la confianza como aquellas cosas de valor que uno posee y deposita en otro para su cuidado, aspectos como la reputación, la amistad, la salud y las inversiones, a la espera de que la otra parte no sacará ventaja en su propio beneficio ni actuará en contra del depositante (Baier, 1986; Zaheer et ál., 1998). De esta definición se desprende que los accionistas, al confiar sus ahorros a las empresas, esperan que estas sabrán invertirlos de forma adecuada, sin dañar sus intereses o, lo que es lo mismo, esperan un comportamiento ético de la empresa (Cortina et ál., 2003).

Gambetta (1988) da otra definición de confianza. Argumenta que confiar en otro significa creer que, cuando surja la oportunidad, la otra parte no tomará decisiones o se comportará de manera que pueda 
dañarnos. Esta confianza cobra relevancia cuando al menos una de las partes es libre de estar en desacuerdo con la otra; libre para evitar un riesgo en la relación y, conscientemente, considera que la relación es una opción atractiva. Basándose en la teoría de los juegos, Gambetta llega a concluir que en la formación de la confianza interviene la realización de un cálculo anterior a la relación, un cálculo sobre la probabilidad de que la organización en que se deposita la confianza hará algo positivo para el inversor. Se creará una situación de confianza cuando la probabilidad de que la otra parte actúe de forma beneficiosa, o al menos no perjudicial, para quien va entrar en la relación sea alta; lo suficiente para que esta considere el comprometerse en alguna forma de cooperación.

Desde este punto de vista, la confianza consiste en hacer frente a la incertidumbre, pero no en forma ciega e inconsciente, sino deliberada y estudiada (Sabel, 1993; Scharpf, 1993). Puesto que se desconoce el comportamiento futuro, la confianza se basa en la teoría individual de que la otra parte se comportará en el futuro en función de su comportamiento actual (Good, 1988).

Para Luhmann (1988), la confianza es la opción de exponerse uno mismo a una situación de riesgo en la cual el daño puede ser mayor que el beneficio a obtener. Esta diferenciación es crucial para entender la fuerza de la confianza ya que, sin confianza, cualquier riesgo estaría dentro de los límites aceptables de la elección racional y, por tanto, esa decisión se basaría en la confianza (Kramer y Tyler, 1996). La confianza permite tomar decisiones de riesgo. La relación con las organizaciones requiere confianza como condición para establecer una relación de inversión, pues esta presupone una situación de riesgo (Luhmann,
1988). La confianza se produce cuando, entre varias posibles decisiones/acciones, se selecciona una concreta, a pesar de que cabe la posibilidad de que la otra parte actúe de modo contrario a lo esperado. La selección se realiza en función de la trayectoria del comportamiento, el conocimiento y las experiencias anteriores con la organización (Zucker, 1986).

Surge en este punto la pregunta acerca de cuál es el modo de producir confianza. Zucker (1986) define tres mecanismos de producción de la confianza. El primero se basa en la experiencia personal de quien deposita la confianza. Cuando se produce una interacción recíproca entre las partes en el corto plazo tal interacción produce seguridad y estabilidad, la cual engendra una propensión hacia la confianza. En las organizaciones, la repetición satisfactoria de tales interacciones a lo largo del tiempo llega a convertir la relación en un compromiso de largo plazo (Arrow, 1984), con la oportunidad para incrementar la cooperación (Good, 1988). Las investigaciones han demostrado que uno de los principales motivos para comprometerse en relaciones de intercambio es el beneficio económico que resulta de este (Peltier y Wesifall, 2000).

El segundo mecanismo se basa en la similitud de características entre las partes. Así, relaciones de familia, amistad o proximidad producen en el depositante una propensión a confiar por la cercanía social (Dore, 1987), la cual enfatiza la sensación de pertenencia y familiaridad (Good, 1988; Guiso et ál., 2005). La justificación teórica para este argumento proviene de la teoría de la categorización social (Tajfel y Turner, 1986; Turner, 1987). De acuerdo con esta teoría, en las relaciones sociales los individuos tienden a clasificar al resto sobre la 
base de ciertos atributos objetivos y observables como la etnia, la profesión o la nacionalidad (Turner, 1987; Charman y Flynn, 2001), con base en estos atributos se perciben a sí mismos como iguales a otros $\mathrm{y}$, por tanto, pertenecientes a un mismo grupo social; o diferentes a otros y, por ello, ajenos a dicho grupo. Estas clasificaciones influyen sobre la confianza porque afectan las creencias y las actitudes de los individuos (Charman y Flynn, 2001). Los individuos tienden a asociar expectativas positivas con las actitudes y los comportamientos sobre aquellos individuos que han clasificado dentro de su mismo grupo social. Dado que la confianza está basada en la existencia de estas expectativas positivas, diversos autores mantienen que se tiende a considerar más dignos de confianza a aquellos individuos que se ha clasificado previamente dentro de la misma categoría social (Brewer y Kramer, 1986; Chattopadhyay, 1999). Según esta línea de razonamiento, resulta más fácil confiar en aquellas organizaciones cercanas a nuestro entorno como, por ejemplo, el banco en el que depositamos nuestro dinero.

El tercer mecanismo se refiere a las características de la propia organización. En este caso la confianza se vincula con el comportamiento de la organización y su relación con el entorno, principalmente el comportamiento de los directivos de la empresa (Pfeffer, 1972). Cuando la dirección tiene buena reputación es más fácil confiar. En este sentido, los códigos de buen gobierno han proporcionado pautas de actuación que pueden llevar a los inversores a conocer y evaluar el comportamiento de los órganos de gobierno de la organización. Los códigos de buen gobierno también han suministrado evidencia de que las organizaciones proporcionan más información valiosa acerca de ellas mismas, sus órganos de gobierno y su comportamiento frente a terceros.

Las organizaciones, de modo análogo a las personas, se forjan un carácter u otro a lo largo de sus vidas. Un carácter por el que se identifican y por el que los demás las identifican. Las decisiones que toman a lo largo de su accionar y que van forjando su carácter pueden convertirse en costumbre de modo que su comportamiento sea predecible. Cuando una organización ha generado las disposiciones o las costumbres requeridas para tomar buenas decisiones, quienes se relacionan con ella pueden esperar que actúe de acuerdo con los fines demandados por la sociedad y, por tanto, llegar a la conclusión de que se trata de una organización digna de confianza (Fukuyama, 1998).

De ahí que lo inteligente por parte de la organización sea incorporar aquellos comportamientos que pueden conducir al cumplimiento de sus fines, ya que de forma inevitable va a tener que decidir a lo largo de su vida cómo actuar para alcanzarlos. El que incorpore cuanto antes pautas de actuación éticas para alcanzar esos fines ahorra energía a la empresa, al evitarle el esfuerzo de cambiar costumbres ya adquiridas que no se adecuan a lo que espera la sociedad (Cortina et ál., 2003). Dicho de otro modo, el cambio continuo de la dirección y las decisiones en las empresas supone incrementar los costos de transacción y agencia de forma que puede llevar a la empresa a incurrir en dificultades financieras y pérdida de confianza.

De esta manera, la confianza genera una espiral que la incrementa (Gambetta, 1988), ya que la cadena de valor de la confianza aumenta con el uso (Putnam, 1994). Además, la confianza se ve ampliada en 
mayor medida cuando es reforzada por un tercero externo a la organización, como demostraron Burt y Knez (1995).

Kramer y Tyler (1996), sugieren que en el desarrollo de una relación entre inversores y organizaciones operan tres tipos de confianza:

- Confianza basada en la disuasión: Se basa en un comportamiento consistente, es decir, los inversores saben que la organización hará lo que ha dicho que va a hacer. En caso contrario se produciría una ruptura en la relación debido a la violación de la relación de confianza.

- Confianza basada en el conocimiento: Parte de que el inversor tiene suficiente información sobre la empresa, la conoce y puede predecir con claridad su comportamiento.

- Confianza basada en la identificación: Se apoya en una relación de empatía con la otra parte, sus deseos e intenciones. Existe una conexión emocional entre las partes de forma que el agente permite actuar a la organización de manera independiente, sin control, pues cree que sus intereses serán respetados, lo que reduce los costos de agencia.

Cuanto más complejas y globales son las empresas y sus entornos sociales, más grandes son los ámbitos en los que los mecanismos de control habituales: capital, órganos de gobierno y normas legislativas pierden eficacia, y gana importancia la obligatoriedad de los estándares morales como la confianza, lo que dejan entrever los códigos de buen gobierno (Wieland, 1993). Por esta razón se desarrolla a continuación la importancia de la confianza entre accionistas y empresa.

\section{Papel de la confianza en la relación principal-agente}

El análisis de los problemas originados por la información, la incertidumbre y la divergencia de intereses en las relaciones económicas entre los distintos participantes en la organización empresarial ha cobrado gran importancia en los últimos años. Esto es debido a la existencia de empresas cada vez más grandes que acuden a los mercados de valores en busca de financiación, lo que ha dado lugar a menores niveles de concentración del accionariado y a la creciente participación de pequeños inversores que buscan mayores niveles de rentabilidad (Granovetter, 1992). Como ya pusieron de manifiesto Berle y Means (1968), las grandes compañías se caracterizan por la dispersión de las acciones entre los inversores, lo que supone una separación entre propiedad y control en la empresa. Este hecho incrementa la posibilidad de que se produzcan conflictos de intereses entre accionistas y directivos, los cuales pueden provocar que las decisiones tomadas por estos últimos no siempre coincidan con el objetivo de los accionistas de maximización del valor, debido a la discrecionalidad de la directiva a la hora de asignar los fondos del inversor (Larner, 1966; Machlup, 1967).

La teoría contractual de la empresa la concibe como un conjunto de contratos que especifican los derechos de propiedad objeto de transacción dentro de la empresa y establecen la forma en la cual se asignarán los costos y las compensaciones entre las partes contratantes, incidiendo así en el comportamiento individual de los participantes de la organización. Esta relación contractual se denomina relación de agencia. A través de ella, una persona, llamada principal, designa a otra, denominada agente, 
para que realice algún servicio en su beneficio; con este propósito, el principal tiene que delegar en el agente cierta autoridad para que pueda tomar decisiones. Cada parte de la relación de agencia perseguirá maximizar su propia utilidad, por tanto es lógico considerar que sus intereses no serán siempre coincidentes, es decir, el agente no actuará en todas las ocasiones de la manera más ventajosa para el principal, lo que provoca un riesgo moral (Salas Fumás, 2002).

En este sentido, el gobierno de la empresa se establece con la finalidad de hacer converger los intereses de los directivos con los de los accionistas y asegurar en última instancia que las empresas se gestionen en beneficio de los accionistas (Donaldson y Preston, 1995) o, en un sentido más amplio, de todos los participantes en el proceso de producción de valor, sean suministradores de capital o deuda, trabajo $\mathrm{u}$ otros bienes y servicios. Por lo tanto, la necesidad del gobierno de la empresa surge a partir del problema de agencia o el conflicto de intereses entre los miembros de la organización y por la existencia de costos de transacción que impiden resolver los problemas de agencia a través de un contrato (Hart, 1995).

Al estudiar el papel de la confianza en las organizaciones, los economistas se han decantado por la posición de calcular la confianza (Williamson, 1993). Las definiciones existentes en este ámbito coinciden en señalar que la confianza está basada en un conjunto de creencias sobre la otra parte (trustee) puestas en la mente del individuo que confía (trustor), denominadas antecedentes o dimensiones de la confianza (Gambetta, 1988; Mayer et ál., 1995; Lewicki et ál., 1998). La mayoría de esas definiciones introducen la confianza como una creencia basada en la ausencia de motivos oportunistas en la otra parte como requisito imprescindible para que esta surja.

Rousseau et ál. (1998) definen la confianza como un estado psicológico que comprende la intención de una parte de aceptar volverse vulnerable frente a la otra, con base en la existencia de expectativas positivas sobre las intenciones y el comportamiento de la otra parte. Debido a que la confianza está basada en estas expectativas positivas, lleva asociada un riesgo en el sentido que implica un aumento de la vulnerabilidad del trustor frente al trustee, porque este último puede no cumplir con dichas expectativas (Mayer et ál., 1995; Portales et ál., 1998; Rousseau et ál., 1998). Por este motivo, algunos autores establecen que el trustor decide asumir más o menos riesgos en su relación con base en un cálculo racional sobre sus posibles ganancias o pérdidas hecho en función de las características del contexto que rodea la relación (Sitkin y Roth, 1993; Mayer et ál., 1995).

El problema es que, aunque todos los autores reconocen la existencia de esta relación entre confianza y riesgo, no se ponen de acuerdo en determinar cuál es el efecto de este último sobre la primera. Así, según Mayer et ál. (1995), el riesgo que percibe el trustor derivado del contexto no afecta su evaluación sobre la confianza del trustee, sino que son dos procesos independientes. Por una parte, el trustor determina el nivel de riesgo que se deriva del contexto en el que se lleva a cabo la evaluación de la confianza del trustee; y, por la otra, evalúa esta confianza. Una vez establecido el nivel de confianza en el trustee, compara este con el nivel de riesgo percibido y actúa en consecuencia: si el nivel de confianza supera al riesgo percibido, el sujeto estará 
dispuesto a asumir riesgos en la relación, de lo contrario no lo hará.

Sin embargo, frente a esta visión, otros autores mantienen que, debido a que no todos los individuos tienen las mismas actitudes y dado que dichas actitudes son desconocidas, el trustor tiene que llevar a cabo la evaluación sobre la confianza del trustee con información limitada, lo que lleva asociado un riesgo. Esto es debido a que no existen ni información ni mecanismos preventivos suficientes para garantizar que la otra parte no va a actuar en su propio beneficio (Portales et ál., 1998).

Desde el enfoque de la teoría de sistemas, Luhmann (1996) desarrolla la función que cumple la confianza. Para él, la confianza es un mecanismo que permite al trustor reducir la incertidumbre a través de la adopción de expectativas específicas sobre el comportamiento futuro del trustee y predecir en alguna medida sus acciones de manera rutinaria, no necesariamente como resultado de un cálculo (Lane y Lubatkin, 1998).

La creencia en la ausencia de motivos oportunistas es lo que la literatura denomina benevolencia, pero no es la única creencia que puede llevar a una parte a confiar en otra pues la confianza se considera un constructo multidimensional (Mayer et ál., 1995; Ganesan y Hess, 1997; Lewicky et ál., 1998). Aunque la doctrina no se pone de acuerdo para establecer cuáles son esas dimensiones, Butler y Cantrell (1984) propusieron cinco componentes específicos de la confianza: integridad, competencia, consistencia, lealtad y cercanía. Siguiendo esta línea de investigación, Burtler (1991) propuso hasta diez condiciones que podían llevar a una persona a confiar en otra, las cuales incluían la integridad, la lealtad y la sinceridad. El modelo propuesto por Sitkin y Roth (1993) incluye una dimensión referida a la habilidad y otra a la congruencia de valores. García-Marzá (2004) postula un modelo de diez dimensiones cuyo cumplimiento llevaría a la confianza entre los individuos (stakeholders) y las organizaciones. Los atributos que comprende son: integridad, credibilidad, justicia, diálogo, transparencia, dignidad, legalidad, compromiso cívico, ecología y responsabilidad.

Partiendo de este modelo, y dadas las características de los accionistas (shareholders) como propietarios de la empresa, las dimensiones por las cuales el agente (trustor) decide confiar en la otra parte (trustee) son: 1) sus expectativas sobre la credibilidad e integridad del otro; 2) su posibilidad de diálogo o comunicación con el otro; 3) sus expectativas sobre la transparencia del otro; 4) su identificación con principios de compromiso social; y 5) sus expectativas de cumplimiento de la legalidad por parte del otro (véase gráfico 1).

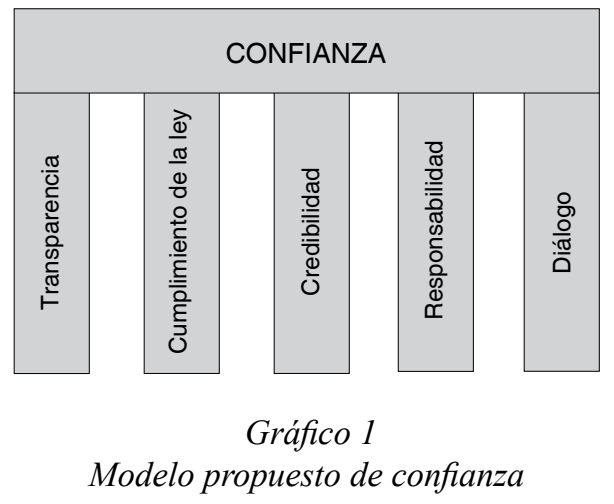

La dimensión de credibilidad se refiere al convencimiento que tiene una parte (el trustor) de que la otra (el trustee) desempeñará con éxito las expectativas depositadas sobre ella y se comportará en forma íntegra aun en circunstancias adversas. Un ejemplo 
de las condiciones que afectan la confianza en esta dimensión es la consecución de resultados (Sitkin y Roth, 1993).

La dimensión del diálogo hace referencia a las posibilidades de comunicación que una parte (el trustor) desea tener con la otra (el trustee) por medio del establecimiento de mecanismos de discusión, participación y consenso. Así, la confianza está basada en las expectativas que una parte (el trustor) tiene sobre la otra (trustee) de que, en caso de conflicto, el trustor se comportará según lo acordado para superar los peligros de los contratos incompletos. Por eso, se propone una adecuada comunicación con el fin de inducir a las partes a adherirse al acuerdo (Pearce et ál., 2000). Tsai y Ghoshal (1998) plantean en su análisis que cuando las dos partes, el trustor y el trustee, comienzan a confiar una en la otra aumenta su predisposición a compartir recursos sin preocuparse de que la otra parte se beneficie en mayor medida. Estos investigadores demostraron que existe una relación positiva entre la percepción de que una parte es merecedora de confianza y la cantidad de recursos intercambiados. Las empresas que disfrutan de una posición de merecedoras de confianza ponen a disposición de los grupos de interés una mayor accesibilidad de recursos, información y conocimientos.

Muy unida a la dimensión del diálogo se encuentra la de la transparencia, es decir, la accesibilidad de la información voluntaria, útil y a tiempo que una de las partes (el trustor) espera que la otra (el trustee) ponga a su disposición para tomar decisiones adecuadas, lo que evita en la medida de lo posible la racionalidad limitada.

La dimensión de compromiso social se refiere al juicio moral que una parte (el trustor) hace sobre la adhesión de la otra (el trustee) a ciertos valores, principios y normas sociales que esta considera deseables (Pérez Díaz, 2001). Estos valores se relacionan con el compromiso y la acción social de la empresa, la contribución al desarrollo local y regional, el respeto a los derechos humanos, la sostenibilidad del medio ambiente y el compromiso con sus empleados. En la medida que las acciones del trustee sean consistentes con su discurso, aumentará la creencia del trustor en su integridad y, por tanto, la confianza en él (Sitkin y Roth, 1993; Butler, 1991). El cumplimiento de esta dimensión significa que no existe ningún comportamiento oportunista por parte de la dirección, lo que produce una reducción de los costos de agencia (Trueman, 1986).

La dimensión de legalidad se refiere a la demostración evidente para una parte (el trustor) de que la otra (el trustee) no incurre en responsabilidad civil ni penal y cumple todas las leyes y las disposiciones legales que la afectan. Esa demostración evidente puede ser la ausencia de sentencias judiciales firmes contra la empresa o su dirección. En concreto en este artículo se va a analizar el cumplimiento de la legislación vigente en materia de gobierno corporativo electrónico.

Estas dimensiones coinciden con lo que la doctrina denomina «confianza basada en el conocimiento» (Robinson, 1996), en contraposición a la «confianza basada en el afecto» en la cual se desarrollan otras dimensiones como la benevolencia (Mayer et ál., 1995).

Como se ha señalado, los accionistas consideran a la empresa digna de confianza cuando cumple con lo acordado. Al ser las empresas gobernadas por personas, la responsabilidad de cumplir con lo acordado 
recae sobre los directivos. Entre ellos y los accionistas se crean contratos morales basados en la confianza. Este contrato moral está en línea con la teoría de la agencia al considerarse como un contrato de largo plazo que especifica de manera amplia los términos de una relación de agencia (García-Marzá, 2004). Esta concepción hace que la confianza se pueda definir a través de variables como los años que llevan los directivos trabajando en la empresa o el puesto que ocupan, el currículo de los consejeros independientes y sus criterios de selección. La confianza se mantiene, desarrolla y refuerza si los órganos de la empresa responden a esta demanda de información y poseen canales de comunicación y participación para todos los grupos de interés. Esta es la razón por la cual las dimensiones descritas se convierten en pilares en los que se apoya la confianza, como se muestra en el gráfico 1.

De acuerdo con lo expuesto, se puede afirmar que para que exista confianza entre accionistas y empresa es preciso que las empresas implementen un canal de comunicación fluido y transparente. En este sentido, Internet puede llegar a ser este canal que fomente el conocimiento de la empresa y el desarrollo de las dimensiones propuestas.

\section{Confianza a través del gobierno corporativo electrónico}

Dado que el entorno es cada vez más competitivo y los avances tecnológicos son más rápidos, el gobierno de la empresa también cambia hacia un modelo estratégico-tecnológico-social en el cual se descentraliza la capacidad de decisión y se refuerza la importancia de la confianza y la disponibilidad de la información.
Gracias a la tecnología, la información puede ser acumulada y administrada para aprovecharla como un capital más. El capital social se entiende como el conjunto de redes, normas y relaciones de confianza que potencia los valores, favorece la coordinación, fortalece los valores compartidos y mejora la reputación, en el marco de un proceso que se autorrefuerza e incrementa (Putnam, 1994). Respecto de los accionistas, esos menores costos se materializan en: menores costos de transacción, menores costos de ruptura de relaciones, mejora de la comprensión de los riesgos y de la resolución de las discrepancias (Rodríguez Fernández, 2003).

El capital social basado en relaciones de confianza puede ayudar a definir un nuevo modelo de gobierno estratégicotecnológico-social de las organizaciones. Entre estos factores, como indican Bueno et ál. (2002), destacan dos. El primero es el aumento progresivo de la diferencia entre el valor de mercado y el valor contable de las organizaciones, en parte provocado por el mayor peso de los intangibles en la economía actual. Y el segundo es la relevancia no solo de los contratos formales, explícitos y pretendidamente completos con los agentes del mercado, como destaca el modelo financiero mercantil de la empresa basado en el objetivo de maximizar el valor para el accionista en el corto plazo (Jensen y Meckling, 1976); sino también de los contratos relacionales o implícitos, basados en la confianza, la lealtad o la ética o el enfoque en los stakeholders ya comentado (Zingales, 2000).

Inkpen y Currall (2004) consideran que la confianza crea un clima que hace posibles las transacciones entre la organización y el mercado. Sin embargo, el desarrollo de la confianza no es fácil por parte del 
trustor sino existe vigilancia y control del comportamiento del trustee. Las decisiones tomadas por el trustee son rápidamente transmitidas al mercado, lo que permite al trustor validar y controlar las acciones de este. Así, la posesión de información clave y el control del flujo de información crean oportunidades empresariales. Pero, como se ha dicho, la confianza no se desarrolla sin vigilancia y control. La tecnología de Internet proporciona esas herramientas de control y vigilancia sobre la empresa que propician el clima de confianza.

En la última década han proliferado los códigos de buen gobierno, es decir, el conjunto de dispositivos, mecanismos y estructuras que determina la forma en que la compañía se dirige y controla; cuya existencia conduce a la obtención de mejores estándares de responsabilidad de la dirección, rendición de cuentas a los accionistas y creación de riqueza (Alonso Almeida, 2007).

Siguiendo las recomendaciones de los códigos de buen gobierno, algunas leyes ya obligan a las empresas a disponer de un portal en Internet a través del cual puedan informar a sus accionistas, a los inversores y al mercado en general acerca de los hechos económicos y de todo acontecer de carácter significativo que se produzcan en relación con la sociedad, para facilitar la participación de los accionistas en el ejercicio de su derecho de información. Para permitir el acceso a la información y ser realmente útil, este portal debe cumplir determinados requisitos técnicos y jurídicos y un requisito de contenidos mínimos (Alba et ál., 2003). Estos se proponen y explican someramente a continuación, sin ánimo de exhaustividad.
1) Requisitos técnicos y jurídicos del portal de Internet

- Página con nombre de dominio registrado. No se exige ni que lleve un sufijo determinado ni que coincida o haga referencia a la denominación social. La utilización de cualquier nombre para la página no facilita al accionista o inversor su búsqueda rápida.

- Acceso rápido y directo a los contenidos por medio de un vínculo directo desde la página inicial de la empresa con la denominación «información para accionistas e inversores». Su visualización no debe requerir más de tres pulsaciones de ratón desde la página principal. Los contenidos deben estar estructurados y jerarquizados, puestos de forma lógica y aparecer en el mapa de la página de la sociedad. De aquí se deduce que esta debe contener de manera obligatoria un mapa de la página de Internet.

- Acceso gratuito. Lo que significa que la empresa no cobre dinero por el acceso a los contenidos, no que la conexión a Internet sea gratuita. Lo que debe ser obligatoriamente gratuito es el acceso a los contenidos.

- Acceso libre. El acceso debe ser libre, es decir, sin necesidad de introducir una clave o una contraseña.

- Información comprensible. Al menos, todas las páginas deben estar redactadas en castellano, con un leguaje sencillo adaptado al inversor medio. Los títulos deben ser claros, entendibles y sin acrónimos.

- Todas las páginas se deben poder imprimir. 
- La información se puede presentar de forma directa o mediante enlaces a las bases de datos que contengan la información. Siempre respetando los requisitos ya señalados.

2) Requisitos de los contenidos (de acuerdo con la agrupación de Muñoz Paredes, 2004)

a) Información general sobre la sociedad:

- Estatutos sociales.

- Capital social y acciones actuales con sus clases, cuadro de evolución, mercados en los que cotiza, calendario de abono de dividendos, emisiones y ofertas públicas de venta (OPV).

- Datos de participaciones significativas y autocartera.

- Ofertas públicas de acciones.

- Hechos relevantes: el texto íntegro comunicado a la Comisión Nacional del Mercado de Valores (CNMV).

- Agenda del inversor con las fechas más relevantes para el accionista respecto de la sociedad.

- Direcciones físicas y electrónicas o números de teléfono a disposición del accionista para comunicarse con la sociedad.

b) Información económico-financiera:

- Cuentas anuales, informe de gestión e informe de auditoría del último ejercicio cerrado y los dos precedentes.

- Información pública periódica que la empresa debe remitir a la CNMV.

- Calificación otorgada por las entidades clasificadoras. c) Gobierno corporativo:

- Junta General: texto íntegro de su reglamento. Convocatoria. Orden del día y propuestas del consejo. Cauces para solicitar la información, delegar el voto o votar a distancia. Información sobre las juntas celebradas en el ejercicio en curso y el precedente.

- Consejo de Administración: composición del consejo y sus comisiones, los vínculos existentes de cada uno de sus miembros con los accionistas. Asimismo, deben publicarse el Reglamento del Consejo y el Reglamento Interno de Conducta.

- Texto íntegro de los pactos parasociales.

- Texto del informe anual de gobierno corporativo.

El portal de la sociedad puede ser el espacio para consignar cualquier hecho de carácter relevante sobre el que el consejo considere conveniente informar y, en especial, las operaciones relacionadas o vinculadas entre la sociedad y sus consejeros, administradores o accionistas, que pudieran aprobarse y fueran significativas.

También algunos códigos recomiendan, para buscar la simetría de la información, que se dé cuenta en este portal de un resumen de los informes emitidos por los principales analistas, bancos de inversión o agencias de calificación que siguen a la sociedad de manera continua.

Para los consejos de administración puede ser una buena oportunidad abrir una vía de diálogo en la página de Internet de la empresa a partir de algún hecho que se desee comunicar. Así, debería haber un canal directo de comunicación bajo la denominación «el consejo informa», por el cual 
los accionistas pudieran comunicarse con el consejo de administración y exponer sus propuestas y dudas y el consejo responder a ellas y explicar lo que está haciendo la empresa para lograr sus objetivos. Para conseguir que los accionistas se sientan confiados y seguros de la relación que mantienen con la empresa deben poder predecir aquello que puede ocurrir en el futuro.

Los aspectos técnicos de Internet, aunque poco desarrollados, se han configurado como esenciales para garantizar el éxito en su uso y utilidad. Así, el concepto «usabilidad» de un sistema de software, introducido por J. Nielsen (1993), tiene dos componentes principales. El primero se refiere al aspecto funcional del sistema, las acciones o las operaciones que este realiza y, el segundo aspecto, cómo los usuarios pueden emplear esa funcionalidad, es aquel que resulta relevante para el presente estudio. Los factores principales que deben considerarse al hablar de usabilidad son la facilidad de aprendizaje, la efectividad de uso y la satisfacción con que las personas son capaces de realizar sus tareas gracias al empleo del producto con el que están trabajando, factores que descansan en las bases del llamado «diseño centrado en el usuario» (Bevan, 2003). El grado de usabilidad de un sistema interactivo es un aspecto relacionado con la interfaz de usuario (Granollers et ál., 2003) que es inversamente proporcional al tiempo que malgastan los usuarios al intentar averiguar qué hace o dónde está determinada función (Bevan, 2003).

La definición «formal» del término usabilidad propuesto por el estándar ISO
13407-11 es: «La medida en la que un producto se puede usar por determinados usuarios para conseguir objetivos específicos con efectividad, eficiencia y satisfacción en un contexto de uso especificado».

Los beneficios que la usabilidad puede aportar a la implementación de sitios en Internet permiten una mayor productividad y una reducción del esfuerzo. La facilidad de uso contribuirá a su «fidelización», pues el visitante volverá y posiblemente recomendará el sitio a sus conocidos y amistades. Si el portal no es «usable» disminuyen la salud, el bienestar y la motivación y se puede incrementar el ausentismo de los accionistas.

Si se centra el interés en la confianza que la empresa desea desarrollar a través de Internet se puede decir que el objeto de la confianza no es solo la empresa sino también el portal de esta y la tecnología de Internet (Jarvenpaa et ál., 2000). El tercer elemento interactúa con la página como si interactuara con la empresa, por lo que la percepción de la confianza en la empresa se desarrollará a través de las interacciones con su portal. Así, la transparencia y la credibilidad de la información de la página de Internet y la confiabilidad de las funciones técnicas que presenta configuran la producción de la confianza a través de la red (véase gráfico 2).

Con el desarrollo del gobierno corporativo electrónico se consigue más transparencia, mayor valor para el accionista, reducción de los costos de agencia y, por tanto, confianza. 


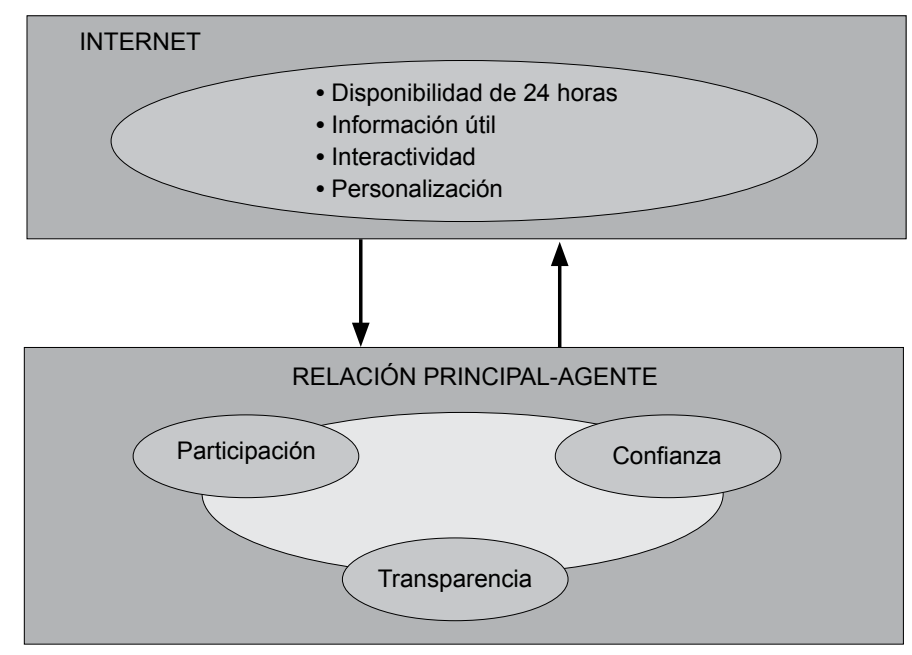

Gráfico 2

Marco de la relación principal-agente a través de Internet

\section{Conclusiones}

Desde el punto de vista de la legislación, el derecho puede concebirse como un mecanismo de implementación de la confianza cuya eficacia viene garantizada por la presencia del Estado (Dreier, 1985); pero este no es el único, el derecho es necesario ya que la confianza tiene mucho que ver con el comportamiento futuro de las empresas y el derecho, con su capacidad de vigilancia y sanción, actúa cuando la empresa no se ajusta «a derecho». Dicho de otra forma, ni la ley ni el mercado son mecanismos suficientes para regular los comportamientos de las organizaciones (García-Marzá, 2004). La comunicación y el diálogo pueden crear confianza activa mediante la apreciación de la integridad de la otra parte (Dellarocas, 2003; Bolton y Katok, 2004). En esta perspectiva, la transparencia se convierte en un factor esencial en el cual la confianza crece en forma proporcional a la disposición de la empresa a responder ante las demandas de información sobre su comportamiento. Información que no debe ser solo económico-financiera, sino que debe incluir aspectos relevantes para todos los grupos con intereses legítimos.

Internet ofrece múltiples posibilidades para que las compañías divulguen más y mejor información financiera, con un costo razonable y hacia una audiencia numerosa y territorialmente dispersa.

En el caso particular del gobierno corporativo, Internet pone a disposición de las compañías múltiples posibilidades para mejorar su imagen y, sobre todo, facilitar la implicación de los accionistas en el control y las prácticas efectivas de buen gobierno. No obstante, ello depende del compromiso real que tengan la compañía y sus accionistas en el desarrollo de un entorno digital que facilite el diálogo y la comunicación entre ambos y así contribuya a fidelizar a los inversores. 
Como se ha puesto de manifiesto, se puede considerar que estamos en el inicio del que podríamos denominar «gobierno corporativo electrónico», cuya aplicación y desarrollo puede plantear, entre otras, ventajas que las empresas deben aprovechar.

En primer lugar, facilita la comunicación entre empresas e inversores, especialmente los accionistas. El portal de la compañía puede utilizarse como un canal de información para que los accionistas, $\mathrm{y}$ los terceros interesados en general, puedan acceder a todo tipo de información relacionada con el devenir del negocio. Además, la empresa puede establecer servicios de alerta que puntualmente faciliten información a los accionistas que se suscriban, y crear perfiles específicos en función de los intereses y el grado de conocimiento que tengan. Puesto que en la comunicación está implícita una acción bidireccional, esto significa que tanto accionistas como terceros podrán comunicarse con la empresa cuando deseen conocer algo de su situación actual o futura. Para ello, la empresa puede poner en Internet a disposición de cualquier interesado un formulario o un correo electrónico para solicitar información. Esta es una primera proposición general para su posterior estudio.

En segundo lugar, se reducen los costos de transacción y se incrementa la oportunidad de acceder a la información corporativa (Williamson, 2002). El derecho de información del accionista obliga a que las empresas pongan a su disposición una serie de datos con carácter previo a la celebración de las juntas de accionistas. La página de la compañía es un medio idóneo para que esta información sea accesible a todos los accionistas de forma rápida, barata y universal (Winter et ál., 2003). Como consecuencia, fomenta la participación de los accionistas en la vida social de la compañía. A través de Internet, las sociedades pueden desarrollar juntas generales por vía telemática, eliminando, entre otras, las barreras geográficas que dificultan o impiden la asistencia, en lo que se puede llamar juntas virtuales. Además, permite una mayor participación de los accionistas en las decisiones de la empresa mediante el voto electrónico.

En tercer lugar, democratiza el acceso a la información corporativa. Existe un riesgo evidente y continuo de que las sociedades que cotizan en bolsa suministren información de forma desigual a los agentes interesados. Ello pone en peligro el principio de transparencia y equidad informativa, necesario para una correcta formación de los precios de mercado de las acciones y un comportamiento eficiente de estos. En términos de confianza, una política de divulgación activa por parte de la empresa que utilice Internet como canal de comunicación puede contribuir a reducir el problema del free rider e incrementar la confianza del accionista en la empresa, especialmente cuando esta cotiza en mercados de capital globales.

Para finalizar, como resulta evidente, un esquema digital de comunicación tiene indudables ventajas para incrementar y facilitar el flujo informativo hacia los inversores, pues evita los efectos nocivos de la divulgación de información selectiva. Además, la evidencia empírica ha constatado la valoración positiva que el mercado realiza de aquellas empresas que aplican las mejores prácticas de buen gobierno. En consecuencia, comunicar rápida, ordenada y verazmente la asunción de prácticas de buen gobierno corporativo y los hechos relevantes de la compañía mejoran 
la imagen y aportan credibilidad a las decisiones que toman las sociedades.

Desde luego, la consecución de estas ventajas está sometida a diversas restricciones. Entre ellas resultan evidentes las de tipo técnico: infraestructura informática, contratación de servicios en la red, seguridad e integridad del sistema; las de índole legal: poco reconocimiento explícito en la legislación societaria y bursátil, efectos sobre la responsabilidad de los administradores, consecuencias jurídicas de nivel transnacional; $y$, en especial, las de tipo estratégico: compromiso real con la transparencia al margen de acontecimientos o situaciones puntualmente favorables o desfavorables.

\section{Referencias bibliográficas}

ALBA, C.; ZUBILLAGA, A. y RUIZ, N. 2003. Educación superior y discapacidad: accesibilidad de las páginas web de las universidades estatales. Comunicación y Pedagogía. N. ${ }^{\circ} 188$, págs. $25-30$.

ALONSO ALMEIDA, M. M. 2007. El gobierno corporativo electrónico. Análisis desde el enfoque de confianza. Madrid: Visión.

ARROW, K. 1984. The economics of agency. Department of Economics. Palo Alto, CAL: Standford University (mimeo).

1974. The limits of the organization. New York: Norton.

BAIER, A. 1986. Trust and antitrust. Ethics. N. ${ }^{\circ}$ 96, págs. 231-260.

BERLE, A. A. y MEANS, G. C. 1968 [1932]. The modern corporation and private property. New York: Harcourt, Brace \& World.

BEVAN, N. 2003. Usability Net methods for user centred design. Human-Computer interaction: Theory and practice (vol.1). Hillsdale, NJ: Lawrence Erlbaum Associates. <http:// www.usabilitynet.org/tools/13407stds. htm>.

BIANCO, W. T. 1994. Trust: Representatives and constituents. Ann Arbor, MI: University of Michigan Press.
BOLTON, G. E. y KATOK, E. 2004. How effective are electronic reputation mechanisms? An experimental investigation. Management Science. Vol. 50, n. ${ }^{\circ} 11$, págs. 1587-1602.

BREWER, M. B. y KRAMER, R. M. 1986. Choice behavior in social dilemmas: Effects of social identify, group size and decision framing. Journal of Personality and Social Psychology, N. ${ }^{\circ}$ 50, págs. 543-549.

BROWN, P. G. 1994. Restoring the public trust. Boston, MASS: Beacon.

BUENO CAMPOS, E.; RODRÍGUEZ SÁNCHEZ, O. y SALMADOR SÁNCHEZ, M. P. 2002. El capital social en la sociedad del conocimiento. Estado del arte y evidencia empírica. XII Congreso Nacional de la Asociación Científica de Economía y Dirección de la Empresa (Acede). Zaragoza.

BURT, R. S. y KNEZ, M. 1995. Kinds of third-party effects on trust. Racionality and Society. N. ${ }^{\circ}$, págs. 255-292.

BUTLER, J. K., Jr. 1991. Toward understanding and measuring condition of trust: Evolution of conditions of trust inventory. Journal of Management. Vol. 17, n. ${ }^{\circ}$ 3, págs. 643-663.

y CANTRELL, R. S. 1984. A behavior decision theory approach to modeling dyadic trust in superiors and subordinates. Psychological Report. ․ ${ }^{\circ}$ 55, págs. 19-28. 
CHARMAN, J. y FLYNN, F. 2001. The influence of demographic heterogeneity on the emergence of cooperative norms in work teams. Academy of Management Journal. N. ${ }^{\circ} 44$, págs. 956-974.

CHATTOPADHYAY, P. 1999. Beyond direct and symmetrical effects: The influence of demographic dissimilarity on organizational citizenship behavior. Academy of Management Journal. N. ${ }^{\circ}$ 42, págs. 273-287.

CORTINA, A.; CONILL, J.; DOMINGO, A. y GARCÍA-MARZÁ, D. 2003. Ética de la empresa. Claves para una nueva cultura empresarial. Madrid: Trotta.

DELLAROCAS, C. 2003. The digitilization of word of mouth: Promise and challenges of on-line reputation mechanisms. Management Science. Vol. 49, n. ${ }^{\circ}$ 10, págs.1407-1424.

DONALDSON, T. y PRESTON, L. 1995. The stakeholder theory of the corporation: Concepts, evidence and implications. Academy of Management Review. N. ${ }^{\circ} 20$, págs. 65-91.

DORE, R. 1987. Taking Japan seriously. Palo Alto, CAL: Stanford University Press.

DREIER, R. 1985. Moral y derecho. En: Garzón Valdés, A. (ed.). Derecho y Filosofía. Barcelona: Alfa.

FUKUYAMA, F. 1998. Confianza. Barcelona: Liberdúplex.

GAMBETTA, D. 1988. Can we trust trust? En: Gambetta, D. (ed.). Trust making and breaking co-operative relations. Oxford: Basil Blackwell.

GANESAN, S. y HESS, R. 1997. Dimensions and levels of trust: Implications for commitment to a relationship. Marketing Letters. Vol. 8, n. ${ }^{\circ}$ 4, págs. 439-448.

GARCÍA-MARZÁ, D. 2004. Ética empresarial del diálogo a la confianza. Madrid: Trotta.
GARMENT, S. 1991. Scandal. The culture of mistrust in American politics. New York, NY: Random House.

GOOD, D. 1988. Individuals, interpersonal relations and trust. En: Gambetta, D. (ed.). Trust making and breaking co-operative relations. Oxford: Basil Blackwell.

GRANOLLERS, T.; LORÉS, J. y PERDRIX, F. 2003. Usability engineering process model. Integration with software engineering. Proceedings of Human-Computer Interaction (HCI) International 2003. Creta.

GRANOVETTER, M. S. 1992. Problems of explanation in economic sociology. En: Nohria, N. y Eccles, R. (eds.). Networks and organizations: Structure, form and action. Boston, MASS: Harvard Business School Press.

GUISO, L.; SAPIENZA, P. y ZINGALES, L. 2005. Cultural biases in economic exchange. Paper. Chicago, ILL: University of Chicago.

HART, O. 1995. Corporate Governance: some theory and implications. The Economic Journal, N. ${ }^{\circ} 15$, págs. 678-689.

INKPEN, A. C. y CURRALL, S. C. 2004. The co-evolution of trust, control, and learning in joint ventures. Organization Science. Vol. 15, n. $^{\circ} 5$, págs. $586-599$.

JARVEENPAA, S. L.; SIRKKA, L.; TRACTINSKY, J. y VITALE, M. 2000. Consumer trust in an Internet store. Information Technology and Management. Vol. 1, n. ${ }^{\circ}$ 1-2, págs. 45-71.

JENSEN, M. C. y MECKLING, W. H. 1976. Theory of the firm: Managerial behaviour, agency costs and ownership structure. Journal of Financial Economics. N. ${ }^{\circ} 3$, págs. 305-360.

KRAMER, R. M. y TYLER, T. R. 1996 (eds.). Trust in organizations. Frontiers of theory and research. London: Sage. 
LANE, P. J. y LUBATKIN, M. 1998. Relative absorptive capacity and inter-organizational learning. Strategic Management Journal. Vol. 19, n. ${ }^{\circ}$ 5, págs. 461-477.

LARNER, R. J. 1966. Ownership and control in the 200 largest non-financial corporations, 1929 and 1963. The American Economic Review. Vol. VI, n. ${ }^{\circ}$, págs. 777-787.

LEWICKI, R. J.; McALLISTER, D. y BIES, R. J. 1998. Trust and distrust: New relationships and realities. The Academy of Management Review. Vol. 23, n. ${ }^{\circ}$ 3, págs. 438-458.

LUHMANN, N. 1996. Confianza. México D. F.: Universidad Iberoamericana.

1988. Familiarity, confidence, trust: Problems and alternatives. En: Gambetta, D. (ed.). Trust making and breaking co-operative relations. Oxford: Basil Blackwell.

MACHLUP, F. 1967. Theories of the firm: Marginalist, behavioral, managerial. The American Economic Review. Vol. LVII, n. ${ }^{\circ} 1$, págs. 1-33.

MAYER, R. C.; DAVIS, J. H. y SCHOORMAN, F. D. 1995. An integrative model of organizational trust. Academy of Management Review. N. ${ }^{\circ} 20$, págs. 709-734.

MILLER, G. 1992. Managerial dilemmas: The political economy of hierachy. Cambridge: Cambridge University Press.

MUÑOZ PAREDES, J. M. 2004. Nuevas tecnologías en el funcionamiento de las juntas generales y de los consejos de administración. Madrid: Civitas.

NIELSEN, J. 1993. Usability engineering. Boston, MASS: AP Professional.

PEARCE, J. L., BRANYICZKI, I. y BIGLEY, G. A. 2000. Insufficient bureaucracy: Trust and commitment in particularistic organizations. Organization Science. Vol. 11, n. ${ }^{\circ}$, págs. 148-162.
PELTIER, J. W. y WESIFALL, J. E. 2000. Dissecting the HMO-Benefits Managers Relationship: What to measure and why. Marketing Health Service. Vol. 20, n. ${ }^{\circ}$, págs. 4-13.

PÉREZ DÍAZ, V. 2001. Organización civil y sociedad civil: el gobierno de las empresas de las economías avanzadas. En: Del Campo, S. (ed.). Perfil de la sociología española. Madrid: Catarata.

PFEFFER, J. 1972. Merger as a response to organizational interdependence. Administrative Science Quartely. N. ${ }^{\circ} 17$, págs. 382-394.

PORTALES, C.; RICART, J. E. y ROSANAS, J. M. 1998. Understanding trust to build strongs relationships in organizations. En: Hitt, M.; Ricart, J. E. y Nixon, D. R. (eds.). Managing strategically in an interconnected world. New York, NY: John Wiley \& Sons.

PUTNAM, R. 2000. La importancia del capital social. Barcelona: Proa.

1994. Para hacer que la democracia funcione. Caracas: Gálac.

ROBINSON, S. 1996. Trust and breach of the psychological contract. Administrative Science Quarterly. N. ${ }^{\circ}$ 41, págs. 574-599.

RODRÍGUEZ FERNÁNDEZ, J. M. 2003. El gobierno de la empresa: un enfoque alternativo. Madrid: Akal.

ROUSSEAU, D. M.; SIM B. B.; BURT, R. S. y CAMERER, C. 1998. Not so different after all: A cross-discipline view of trust. Academy of Management Review. Vol. 23, n. ${ }^{\circ}$ 3, págs. 393-404.

SABEL, C. F. 1993. Constitutional ordering in historical context. En: Scharpf, F. W. (ed.). Games in hierarchies and networks. Boulder, COL: Westview. 
SALAS FUMÁS, V. 2002. El gobierno de la empresa. Colección de Estudios Económicos de La Caixa N. ${ }^{\circ} 29$. Barcelona: Fundación La Caixa.

SCHARPF, F. W. 1993. Coordination in hierarchies and networks. En Scharpf, F. W. (ed.). Games in hierarchies and networks. Boulder, COL: Westview.

SITKIN, S. B. y ROTH, N. L. 1993. Explaining the limited effectiveness of legalistic "remedies" for trust/distrust. Organizational Science. N. ${ }^{\circ}$ 4, págs. 467-392.

TAJFEL, H. y TURNER, J. C. 1986. The social identity theory of intergroup behaviour. En: Worchel, S. y Austin, W. (eds.). Psychology of inter-group relations. Chicago, ILL: Austin Nelson Hall.

TRUEMAN, B. 1986. Why do managers voluntarily release earnings forecast? Journal of Accounting and Economics. N. ${ }^{\circ}$ 8, págs. 53-72.

TSAI, W. y GHOSHAL, S. 1998. Social capital and value creation: The role of intrafirm networks. Academy of Management Journal. Vol. 41, n. ${ }^{\circ} 4$, págs. 464-476.

TURNER, J. C. 1987. Rediscovering the social group. A social categorization theory. Oxford: Basil Blackwell.
WIELAND, J. 1993. Formen des Institutionalisierung von Moral in amerikanischen Unternehmen. Stuttgart: Haupt.

WILLIAMSON, O. E. 2002. The theory of the firm as governance structure: From choice to contract. Journal of Economic Perspectives. Vol. 16, n. ${ }^{\circ}$ 3, págs. 171-195.

1993. Calculativeness, trust and economic organization. Journal of Law and Economics. N. ${ }^{\circ}$ 34, págs. 453-502.

WINTER, S. J.; SAUNDERS, C. y HART, P. 2003. Electronic window dressing: Impression management with websites. European Journal of Information Systems. Vol. 12, n. ${ }^{\circ}$ 4, págs. 309-322.

ZAHEER, A.; McEVILY, B. y PERRONE, V. 1998. Does trust matter? Exploring the effects of inter-organizational and interpersonal trust on performance. Organization Science. N. ${ }^{\circ}$ 9, págs. 141-159.

ZINGALES, L. 2000. In search of new foundations. Journal of Finance. N. ${ }^{\circ}$ 55, págs. 1623-1653.

ZUCKER, L. G. 1986. Production of trust: Institutional sources of economic structure. En: Staw, B. M. y Cummings, L. L. (eds.). Research in organizational behaviour. Greenwich, CT / London: JAI Press. 